\title{
Adverse Event MedDRA System Organ Class
}

National Cancer Institute

\section{Source}

National Cancer Institute. Adverse Event MedDRA System Organ Class. NCI Thesaurus. Code C117385.

Adverse event term related to the highest level of the Medical Dictionary for Regulatory Activities (MedDRA) terminology, and distinguished by anatomical or physiological system, etiology, or purpose. 